\section{La prevención de la mortalidad por dengue: un espacio y un reto para la atención primaria de salud}

\author{
Eric Martínez Torres ${ }^{1}$
}

Palabras claves: dengue, fiebre dengue hemorrágica, organización y administración.

\footnotetext{
Subdiercción de Docencia de Postgrado. Instituto de Medicina Tropical Pedro Kourí, Centro Colaborador OPS/OMS para el Estudio del Dengue y su Vector, Ciudad de La Habana, Cuba. La correspondencia debe enviarse al autor a: Instituto de Medicina Tropical Pedro Kourí, Autopista Novia del Mediodía km 612/2, Apartado postal Marianao 13, Ciudad de La Habana, Cuba. Correo electrónico: eric. martinez@infomed.sld.cu
}

El dengue es la enfermedad viral trasmitida por vectores más extendida en el mundo y constituye uno de los mayores retos de la salud pública en el presente siglo (1). En la Región de las Américas, el número de casos de dengue ha aumentado sostenidamente durante los últimos 25 años y este aumento se interpreta como una falla de las políticas de salud pública (2). Durante el año 2002 se notificaron más de 1 millón de casos y en el año 2005 se apreció un discreto aumento respecto a los dos años precedentes. ¿Qué sucederá en los años 2006 y siguientes?

La Organización Panamericana de la Salud (OPS) ha desarrollado una estrategia de intervención integrada que ha demostrado ser eficaz en los países donde se ha incorporado al trabajo de prevención y control. No obstante, la posible introducción o reintroducción de nuevos serotipos, así como la circulación de cepas de mayor virulencia en poblaciones que cuentan con un número considerable de personas susceptibles y tienen una infestación moderada o elevada con el mosquito vector Aedes aegypti, apuntan a que en el futuro habrá nuevas epidemias (3).

El dengue es una sola enfermedad que se expresa de diversas maneras $\mathrm{o}$, mejor dicho, lo hace con diversos grados de intensidad aun en una misma epidemia. Una forma es la fiebre indiferenciada, frecuente en niños, y otra es la fiebre asociada con mialgias, cefalea y dolor retroorbitario, leucopenia frecuente y exantema, la cual se presenta en aproximadamente la mitad de los casos. Esta última forma se conoce como fiebre por dengue o dengue "clásico" y en ocasiones se acompaña de petequias $\mathrm{u}$ otras hemorragias menores, aun cuando no se observe una reducción importante del número de plaquetas.

La otra forma clínica reconocida es el dengue hemorrágico/síndrome de choque por dengue (DH/SCD), cuya alteración principal y característica es la extravasación clínicamente importante de plasma, en la cual el enfermo tiene una etapa febril indistinguible de la del dengue y evoluciona posteriormente con trombocitopenia ( $\leq 100000$ plaquetas $\times \mathrm{mm}^{3}$ ), hemoconcentración, derrames serosos (pleural, ascítico o pericárdico), hipotensión y choque, así como hematemesis y otras hemorragias de consideración, todo lo cual coloca al paciente en riesgo inminente de morir (4). En los países de Asia sudoriental, la inmensa mayoría de los casos de DH/SCD notificados ocurren en niños; no obs- 
tante, en las Américas las epidemias de dengue hemorrágico han afectado a niños y a adultos, y en ocasiones han predominado estos últimos, como en Brasil (5).

La mortalidad vinculada con una epidemia de dengue puede estar relacionada con diversas características del virus y el hospedero, como la patogenia de la cepa viral circulante y las particularidades inmunitarias de la población, pero sobre todo con el nivel de preparación del sistema de salud para hacer frente a estas contingencias. Ningún país está exento de tener casos graves, pues aun en Europa hay personas que regresan de zonas endémicas con distintas formas clínicas de dengue, incluso el dengue hemorrágico (6-7). Australia, después de un siglo sin casos mortales, informó en 2005 de dos adultos fallecidos por dengue (8).

Tan importante como evitar la transmisión del dengue es la preparación de los sistemas de salud para atender adecuadamente a los enfermos y evitar su muerte. Se debe aspirar a no tener epidemias, pero, si ocurren, se debe lograr que no haya fallecidos. Un buen administrador de salud es capaz de salvar más vidas durante una epidemia de dengue que los médicos e intensivistas. Esto de debe a que el tratamiento individual de cada enfermo no puede aplicarse adecuadamente si no está enmarcado en un conjunto de medidas organizativas y de capacitación que deben ejecutarse en cada hospital o centro de atención primaria.

Con el presente trabajo se pretende contribuir a reducir la mortalidad por dengue mediante la desmitificación del tema de la aparente falta de tratamientos para esta enfermedad y la aclaración de algunos errores frecuentes. Además, se describen los componentes con que debe contar todo sistema de salud para enfrentar las epidemias de dengue, particularmente en la atención primaria de salud (APS) y en la comunidad, por ser el escenario donde se pueden evitar o reducir al mínimo las muertes durante una epidemia.

\section{MITOS Y ERRORES}

\section{Mitos}

- El dengue no tiene tratamiento.

Con relativa frecuencia se repite esta afirmación, incluso en publicaciones internacionales (9). Es cierto que no se dispone de un fármaco antiviral que disminuya rápidamente la viremia ni se dispone aún de un medicamento o anticuerpo monoclonal capaz de bloquear los mecanismos fisiopatogénicos que conducen al choque, a las grandes hemorragias y al daño multiorgánico. Pero la ca- rencia de este tipo de tecnología tangible puede ser sustituida por la aplicación eficiente de un conjunto de conocimientos que permite clasificar al paciente según sus síntomas y la etapa de la enfermedad, así como reconocer precozmente los signos que anuncian la gravedad del cuadro clínico y decidir a tiempo las conductas terapéuticas más adecuadas.

- Se necesita una abundante reserva de sangre, de sus derivados y de recursos avanzados para evitar la muerte de los enfermos con dengue.

Esto no es así, ya que cada vez se hace menos frecuente la transfusión de plaquetas. No se ha evaluado críticamente su indicación (10) ni se ha demostrado la obligada relación entre la trombocitopenia y los sangrados en una enfermedad en que las hemorragias son multicausales (11-13). No son muchos los casos hemorrágicos que necesitan transfusiones de eritrocitos o de sangre total y una gran parte de las hemorragias pueden prevenirse si el choque por dengue se trata precoz y adecuadamente con soluciones cristaloides $(14,15)$. Ni siquiera resulta imprescindible el tratamiento con albúmina humana o coloides sintéticos (16).

- La única o principal función de los servicios de APS durante una epidemia de DH/SCD es el control del mosquito para evitar la transmisión de la infección y, si acaso, brindar tratamiento sintomático a los casos de "dengue clásico".

La importancia del trabajo del personal sanitario (médicos, enfermeros y técnicos) de la APS es inestimable, como reconoce un documento normativo publicado por la Organización Mundial de la salud (OMS) (17). Además de lo ya expuesto, la APS cumple la importante tarea de educar al paciente y a sus familiares en medidas de autocuidado y en la observación de signos que pueden indicar el agravamiento de los enfermos. La atención médica diaria y frecuente permite identificar precozmente a los enfermos que pueden evolucionar hacia la forma grave, aun cuando todavía no cumplan con todos los criterios de DH/SCD. Esto permite adelantar el inicio de la reposición de líquidos antes de que sean evidentes el choque y las demás complicaciones. Los médicos intensivistas que han atendido a enfermos durante una epidemia de DH/SCD coinciden en que lo que determina el pronóstico final del paciente es su diagnóstico y tratamiento iniciales y su estado al llegar a la unidad de cuidados intensivos, y no la cantidad de recursos empleados después.

A esos mitos se asocian con frecuencia algunos errores que hacen que el administrador de salud aplique esquemas organizativos y normas de tratamiento tradicionales que no son los adecuados -y ni siquiera los correctos-durante una epidemia de dengue. 


\section{Errores}

- Las formas graves de dengue se presentan solamente en personas pobres y menesterosas.

Este error puede llevar al administrador de salud a organizar la capacitación solamente de aquellos médicos y enfermeros de los servicios públicos adonde acude el sector más pobre de la población y dejar de capacitar a los profesionales del sector privado. En Recife, Pernambuco, Brasil - por citar un ejemplo-, durante la epidemia por virus del dengue (serotipo 3) de 2002, la mayoría de los fallecimientos ocurrieron en hospitales privados (18). En todos los países de la Región han fallecido o se han presentado casos muy graves entre médicos, enfermeros, políticos, empresarios, artistas, periodistas y demás representantes de las capas sociales, económicas y culturales más elevadas, lo que demuestra que el dengue no reconoce las diferencias sociales.

- Las complicaciones del dengue se presentan durante el acmé febril de la enfermedad, por lo cual a partir de la caída de la fiebre no es necesario vigilar los signos que anuncian peligro.

En realidad, en el dengue ocurre todo lo contrario: el período crítico coincide con la defervescencia (19). Habitualmente durante la caída de la fiebre, o poco después, se constata una considerable hemoconcentración y se producen derrames en las cavidades serosas, lo cual hace que la extravasación de plasma tenga consecuencias clínicas importantes. A continuación pueden aparecer hipotensión arterial, bajo gasto cardíaco, taquicardia, taquisfigmia, cianosis distal y choque. Todo esto es evitable si la organización de la atención médica establece la observación clínica del enfermo en quien se sospecha dengue - especialmente después de la caída de la fiebre - mediante la vigilancia y la búsqueda activa de signos que anuncian a tiempo el deterioro clínico.

- Los pacientes con "dengue clásico" no suelen tener complicaciones; solamente las sufren los que tienen dengue hemorrágico.

Lo anterior se debe a una clasificación superficial y demasiado temprana de los enfermos con dengue, en la que se atribuye una buena evolución a la enfermedad ("la fiebre por dengue es molesta pero no mata") y se desconoce que con esa forma clínica pueden asociarse con relativa frecuencia alteraciones de la función hepática, miocarditis u otras cardiopatías, así como manifestaciones neurológicas por afectación del sistema nervioso central. Al inicio de la enfermedad no es posible saber qué pacientes evolucionarán mal y llegarán a tener DH/SCD, ya sea con o sin hemorragias (20). Por lo tanto, durante una epidemia todos los enfermos en quienes se sos-

\section{CUADRO 1. Signos de alarma en pacientes con dengue}

Clínicos

1. Dolor abdominal intenso o mantenido

2. Vómitos muy frecuentes y abundantes

3. Descenso brusco de la temperatura, que puede llegar a la hipotermia, con decaimiento excesivo y, a veces, lipotimia

4. Irritabilidad, somnolencia o ambos

De laboratorio

1. Aumento progresivo del hematocrito

2. Descenso progresivo de las plaquetas

peche dengue deben recibir atención médica y educación sanitaria y mantenerse en observación durante la etapa febril y al menos 48 horas después.

- Se debe esperar a que el enfermo cumpla los cuatro criterios de la definición de caso de DH/SCD, según la OMS (fiebre, algún sangrado, trombocitopenia de $<100000 \times \mathrm{mm}^{3}$ y hemoconcentración u otro signo de extravasación de plasma), para iniciar el tratamiento de reposición de líquidos por vía intravenosa.

Un enfermo de dengue puede presentar signos de alarma que anuncian que el choque es inminente (cuadro 1) cuando aún su cuadro clínico no cumple los criterios para clasificarlo como caso de dengue hemorrágico (21). Es por eso que el administrador de salud debe exigir al personal médico y paramédico a su cargo que cumpla cabalmente las pautas para el diagnóstico, tratamiento y seguimiento de los casos de dengue publicadas por la OMS $(22,23)$ y la guía publicada por la OPS $(24)$, con el objetivo de poder aplicar el tratamiento tempranamente y evitar el fallecimiento de los enfermos. Una vez recuperado el enfermo, el médico podrá aplicar la definición de caso y la clasificación de la OMS que establece grados de gravedad (del I al IV), las cuales tienen un claro objetivo epidemiológico.

\section{TEORÍA Y PRÁCTICA}

Los aspectos organizativos de la atención médica durante una epidemia de dengue están esbozados en documentos de la OMS. En ellos se menciona en particular la necesidad de disponer de protocolos específicos para el diagnóstico clínico y el tratamiento de los casos de DH/SCD, la divulgación de información entre los trabajadores de la salud, la programación de adiestramientos a médicos, enfermeros, estudiantes y técnicos, la dotación 
de recursos materiales (tanto de medicamentos como de otros suministros) y la coordinación de la investigación durante las epidemias (25).

Algunos países de la Región han incorporado en sus respectivos programas nacionales de prevención y control del dengue los aspectos organizativos de la atención médica durante epidemias (26-28) o sus autoridades de salud han emitido documentos normativos específicos para el diagnóstico y el tratamiento de la enfermedad, con el fin de evitar o disminuir la mortalidad (29-33). Aunque todos estos documentos toman en cuenta el tratamiento ambulatorio del dengue, el esquema organizativo tiene al hospital y a su servicio de urgencias como centro de todas las acciones de atención médica y no utilizan adecuadamente las posibilidades que ofrece el subsistema de APS.

En 1995, la OPS publicó un documento normativo dirigido a toda la Región que contenía elementos organizacionales y proponía un flujo de atención a pacientes que ha demostrado su eficacia y aún hoy mantiene su vigencia (24). Más recientemente, a partir de la experiencia en Puerto Rico, Rigau y Clark desarrollaron el tema de cómo responder a una epidemia de dengue, optimizar la atención a los pacientes y organizar la educación médica (34).

Sin embargo, pocas veces los documentos normativos se adecuan a las características propias de cada país, ciudad e institución sanitaria o se elaboran con carácter prospectivo. Por lo tanto, su aplicación no siempre es la correcta y no se puede evaluar el impacto de esas medidas en la disminución de la mortalidad.

Por otra parte, el carácter abrupto de las epidemias de dengue y de DH/SCD impone a los sistemas de salud retos adicionales relacionados con el flujo de los enfermos dentro del propio sistema, los exámenes de laboratorio que deben realizarse con urgencia, el seguimiento de los pacientes durante los días que dure la enfermedad, así como la realización de prontuarios que reflejen adecuadamente la evolución de la enfermedad y el examen físico realizado en las diferentes consultas (35). Esto ha llevado a algunos autores a considerar que ninguna unidad de salud está preparada para enfrentar un reto de ese tipo y magnitud (36).

La práctica es la principal fuente de aprendizaje. A continuación se mencionarán brevemente algunos casos particulares.

\section{- Las epidemias cubanas}

En 1981, Cuba fue el primer país de la Región que sufrió una epidemia de DH/SCD (serotipo 2). Apenas en cuatro meses se notificaron más de 360000 casos de dengue, 30000 de los cuales tu- vieron algún tipo de hemorragia y 10000 fueron casos graves de DH con o sin choque $(37,38)$. El desconocimiento de la enfermedad por parte del personal médico fue paliado por un conjunto de orientaciones para la atención de los enfermos con dengue, emitidas por un comité ad hoc. Por su parte, la ausencia de un plan específico para esta contingencia fue suplida por la aplicación del modelo de asistencia de la salud pública cubana en períodos de lucha antiepidémica, la cual se basa en el aseguramiento de la cobertura en servicios de urgencia y unidades de hospitalización, el aumento del número de camas disponibles, el empleo de criterios amplios de ingreso hospitalario, así como en otras acciones novedosas, como la observación clínica continua por estudiantes de medicina y de enfermería y la creación de unidades de atención al paciente grave (que posteriormente se convirtieron en las llamadas unidades de terapia intensiva) (39).

La tasa de letalidad fue la más baja alcanzada hasta ese momento en una epidemia de dengue hemorrágico pero, a pesar de todo, hubo 158 fallecidos - entre ellos 101 niños $(37,38)$ - y los gastos ascendieron a U\$S 103 millones (40). Una gran parte de esos gastos correspondieron a la asistencia médica. Se habilitaron 17218 camas dedicadas a la epidemia, 39,8\% de las cuales eran extrahospitalarias. No obstante, quedó estructurada una estrategia - que de una manera u otra ha sido de utilidad a casi todos los países de la Región- basada principalmente en la capacitación y la organización de los recursos humanos y en el reordenamiento de los recursos materiales disponibles $(41,42)$.

La segunda epidemia de dengue hemorrágico en Cuba ocurrió en Santiago de Cuba en 1997 (serotipo 2) (43). En esta ocasión se aplicaron algunos métodos ya empleados en 1981 —como la hospitalización precoz, el aumento de la disponibilidad de camas y la utilización de los cuidados intensivos para todo caso de DH/SCD - a lo cual se sumó la visita a los hogares y a la comunidad con el doble objetivo de controlar el vector y de buscar activamente los casos clínicos. En esta tarea participaron activamente los estudiantes de medicina y de enfermería.

Algunas semanas después de iniciada la epidemia resultó imposible mantener este esquema de atención centrado en el hospital y fue necesario capacitar a todos los médicos y enfermeros de APS de esa provincia, así como a los estudiantes de medicina y de enfermería, para pasar a un segundo escalón en la estrategia: el ingreso en el hogar. Para ello fue necesario fortalecer ese subsistema y establecer un enlace fluido con el nivel secundario de atención.

Esta estrategia dio buenos resultados y se desarrolló ampliamente durante la tercera epidemia de dengue ocurrida en 2001-2002 en la Ciudad de 
La Habana (serotipo 3). En esa ocasión, el ingreso en el hogar se decidió precozmente y se logró dar esa atención a varios miles de pacientes febriles (con visitas del médico y del enfermero de familia a sus hogares); ninguno de estos enfermos falleció (44). El ingreso en el hogar preveía la utilización obligatoria de mosquiteros y el control del vector en las áreas domiciliares y peridomiciliares. Durante la etapa inicial de esta epidemia fallecieron tres pacientes, todos adultos (45).

\section{- La epidemia salvadoreña en 2000}

El Salvador tuvo su primera gran epidemia de dengue hemorrágico en el año 2000 (serotipo 2) y en su ayuda acudieron equipos sanitarios de Cuba y México. Durante la semana epidemiológica 38 se notificó el mayor número de enfermos (2 883), con una elevada letalidad de $10 \%$ en los casos con DH/SCD (46). El diagnóstico de la situación realizado en ese momento demostró que había problemas para reconocer tempranamente la enfermedad y definir los casos; la clasificación de los pacientes era inadecuada; no se reconocían oportunamente los signos de alarma; se indicaban estudios complementarios innecesarios (lo que provocaba la saturación de los laboratorios); había congestionamiento en los hospitales y los servicios de urgencia; no se contaba con criterios estandarizados de tratamiento, y el seguimiento de los pacientes era inadecuado. Hasta ese momento habían fallecido 32 niños con dengue.

El equipo médico multinacional, conjuntamente con profesionales salvadoreños, elaboró una propuesta de estrategia para la asistencia médica durante la epidemia que fue aceptada por las autoridades nacionales de salud. Esta estrategia contemplaba la unificación de los criterios de diagnóstico y de tratamiento, la capacitación de los profesionales locales mediante un documento basado en las guías de la OPS para la prevención y el control del dengue en las Américas (24), el fortalecimiento de la red de servicios y el desarrollo del nivel primario (creación de las unidades de dengue). Mediante visitas a 30 hospitales y unidades de APS se logró capacitar a 3500 médicos, enfermeros y otros trabajadores de la salud en solo 72 horas y se reorganizó la atención médica. El resultado inmediato fue que no fallecieron más niños por dengue en todo el país y solamente murió una mujer joven que tenía dengue y miocarditis.

Se estableció la búsqueda activa de enfermos sospechados de tener dengue en los domicilios, con la participación de estudiantes universitarios, enfermeros y promotores de salud de la comunidad que habían recibido un adiestramiento previo. Durante la primera semana de la pesquisa, dúos de trabajo compuestos por una persona con formación sa- nitaria y un integrante de la comunidad visitaron 60000 viviendas y examinaron en sus hogares a más de 100000 personas (80\% de ellas menores de 15 años). Se detectaron más de 5000 personas con fiebre, $80 \%$ de las cuales tenían síntomas o signos de dengue. A estas personas se les remitió al hospital o se les indicó tratamiento en el hogar, según el caso. A todas se les explicó cuáles eran los signos de alarma que debían vigilar durante la evolución de la enfermedad. El seguimiento en el hogar de estos pacientes contribuyó a la disminución del número de enfermos atendidos en los hospitales y a consolidar el logro de no tener más fallecidos por dengue (46).

¿Cómo pudo detenerse la mortalidad de forma tan inmediata? Entre los principales factores que influyeron en este logro se encuentran la unificación de criterios para el diagnóstico y el tratamiento y la prioridad que se les dio a estos enfermos en todos los niveles del sistema nacional de salud. Asimismo, quedó validada la clasificación práctica de los pacientes con dengue durante una epidemia, recomendada en las guías de la OPS, y se demostró la utilidad del flujo de atención a pacientes que proponen dichas guías. En ambos casos, se otorga la mayor importancia a la identificación de signos de alarma para iniciar precozmente la reposición de líquidos por vía intravenosa y prevenir el choque (24).

¿Por qué morían esos niños? Una investigación realizada por el autor, dos infectólogos pediatras salvadoreños y la patóloga del Hospital Nacional de Niños Benjamin Bloom permitió conocer que $20(83 \%)$ de los 24 niños que fallecieron durante las primeras 72 horas de hospitalización habían presentado choque prolongado o recurrente al cual sucedieron otras complicaciones, como hemorragias masivas (casi siempre gastrointestinales y respiratorias), coagulación intravascular diseminada, síndrome de dificultad respiratoria por edema pulmonar no cardiogénico y fallo de múltiples órganos como resultado del síndrome de hipoperfusión/ reperfusión. O sea, más que complicaciones del dengue, las muertes infantiles se debían a complicaciones del choque, por lo que puede afirmarse que al prevenir el choque se previenen las grandes hemorragias y los restantes componentes del cuadro de extrema gravedad.

Cabe señalar que los cuatro niños en quienes no se encontraron signos de choque, aunque sí grandes hemorragias desde su ingreso, llegaron al Hospital Bloom referidos de otros hospitales del país, por lo que no se puede asegurar que no hubieran tenido signos de choque en algún momento de su evolución anterior a la hospitalización en el centro de referencia.

En general, algunos enfermos de dengue presentan formas clínicas con predominio visceral (26) y 
signos de gravedad $-\mathrm{o}$ fallecen - por afectaciones neurológicas, miocárdicas o hepáticas, aunque también pueden presentar grandes hemorragias digestivas, al parecer no precedidas por el choque (34). Estas formas clínicas, a las que algunos investigadores se refieren como manifestaciones inusuales del dengue, no siempre tienen un desenlace mortal -al menos en niños (47) - y son de difícil o imposible clasificación de acuerdo con los criterios actuales de la OMS (48). Hasta ahora, estas formas de presentación no han sido frecuentes y, en ocasiones, coexisten con otra enfermedad o afectación del paciente. Es necesario identificar los signos clínicos más precoces y las alteraciones más tempranas en los resultados del laboratorio que permitan dirigir la atención de urgencia a los casos de peor pronóstico. El seguimiento adecuado de cada enfermo permitirá iniciar el tratamiento correcto y oportuno para evitar el fallecimiento de una buena parte de los pacientes.

\section{- La epidemia de Río de Janeiro en 2002}

En los años 2001 y 2002 el virus del dengue (serotipo 3) se introdujo en Brasil y causó una epidemia que ha sido la más grave hasta el momento en ese país por el elevado número de casos de dengue y de DH/SCD. De las casi 2500 personas con $\mathrm{DH} / \mathrm{SCD}$, fallecieron 97 (49). Esta epidemia fue especialmente intensa en el estado de Río de Janeiro ( 1705,5 casos por 100000 habitantes), en particular en el municipio de Río de Janeiro, donde la tasa de enfermedad fue de 3175 por 100000 habitantes.

De los 111 fallecidos presuntamente de dengue, 63 se confirmaron como dengue (15 casos por criterios clínico-epidemiológicos y 48 casos confirmados mediante análisis serológico, reacción en cadena de la polimerasa, inmunohistoquímica o aislamiento viral). De ese grupo, se estudiaron los expedientes clínicos de 45 casos que habían estado hospitalizados y se realizaron entrevistas a sus familiares con el objetivo de determinar en cuáles de ellos se hubiera podido prever y evitar la muerte (50).

Se solicitó atención médica al inicio de la enfermedad (hasta el 3. ${ }^{\text {er }}$ día) en $62,2 \%$ de los casos. De las 77 visitas médicas realizadas, solamente en $35,1 \%$ se orientaron medidas de hidratación y solo en 5,2\% se informaron los signos de alarma. Según los familiares, 95,2\% de los pacientes que luego fallecieron presentaron al menos un signo de alarma y 48,8\% tenían enfermedades previas que podían empeorar el pronóstico. En 58,1\% de los casos, los entrevistadores consideraron que el fallecimiento pudo haberse evitado, lo que coincidió con lo observado en la revisión de los prontuarios clínicos (50).

Durante esa epidemia, el hospital Municipal Lourenço Jorge notificó el mayor número de casos aislados sospechados de tener dengue (16 769, aproximadamente $10 \%$ del total de los casos notificados en el municipio de Río de Janeiro). Durante el período epidémico se observó un incremento promedio de 110 casos por día, por lo cual se ensayaron sucesivamente varios tipos de flujo de atención:

a) el que se prestaba habitualmente en el departamento de urgencias durante el período previo a la epidemia, hasta que el excesivo número de casos con hemorragias u otra manifestación clínica que requería mayor cuidado médico llevó al siguiente esquema

b) para reducir el tiempo de espera y agilizar la atención de los pacientes se estableció una central de atención en el auditorio del hospital, donde un equipo multidisciplinario recibía a los pacientes, ofrecía orientaciones generales sobre la enfermedad y destacaba la importancia de la hidratación y de los signos de alarma, mientras otro equipo se ocupaba de los procedimientos clínicos, tanto diagnósticos como terapéuticos

c) se suspendieron las consultas y la atención ambulatoria para utilizar todo el espacio físico y todos los profesionales de la salud del hospital en la atención exclusiva de los casos con dengue; en esa área de servicios externos del hospital se tomaban también las muestras para los exámenes de laboratorio y se ingresaba a los pacientes por unas horas para observar su evolución y rehidratación (36).

En el referido hospital municipal carioca fallecieron 12 pacientes ( 8 murieron antes de las 6 horas de su llegada al hospital), la mayoría de los cuales se presentaron con signos de choque o con un cuadro clínico próximo al choque. Fue necesario ajustar todo el flujo de atención del hospital para enfrentar una situación de libre demanda de la población que buscaba preferentemente los servicios de emergencia (36), pues al parecer no disponían de otra cobertura o no tenían confianza en otra unidad de salud que no fuera el hospital.

\section{¿QUÉ HACER?}

No tener mortalidad por dengue o reducirla al máximo depende principalmente de la calidad de la atención médica y del acceso oportuno de la población a esa atención médica que, sin dejar de ser curativa, tiene que cumplir funciones de prevención y promoción, especialmente mediante la educación para la salud.

En Tailandia se logró reducir la mortalidad por dengue hemorrágico a 0,2\% en 1999 gracias a la aplicación de un programa de desarrollo de capacidades para la atención de los pacientes con $\mathrm{DH} /$ 
SCD. Este programa comenzó por establecer una política de salud para esta enfermedad, la cual se tradujo en un plan de prevención y control (51).

Algunas de las actividades de ese plan fueron:

a) crear un comité de expertos para analizar las causas de muerte por DH/SCD en todo el país y a partir de los resultados desarrollar las guías nacionales para el diagnóstico y el tratamiento de los pacientes

b) divulgar el contenido de esas guías mediante talleres, conferencias y seminarios e incorporarlas en el currículo de la Escuela de Medicina, Enfermería y Salud Pública

c) proveer el equipamiento mínimo necesario para el diagnóstico y el tratamiento del DH/SCD e impulsar la creación de "áreas de dengue" a distintos niveles del Sistema Nacional de Salud

d) crear una red nacional de dengue en función del sistema de atención y referencia de casos

e) realizar investigaciones, tanto biomédicas como sociales, particularmente en relación con la educación de los pacientes y sus familiares sobre el curso natural de la enfermedad y la atención en el hogar (51).

La capacitación del personal médico y de enfermería resulta fundamental para ofrecer una atención de calidad a los pacientes con dengue, pero esto no basta, pues durante el enfrentamiento de las epidemias el modelo de atención es determinante (35). El tratamiento individual de cada enfermo debe formar parte de un conjunto de medidas organizativas y de capacitación que se deben aplicar en los hospitales, centros de atención primaria y la comunidad. Entre esas medidas organizativas se debe contemplar el adiestramiento de todo el personal médico y de enfermería, la educación de la población, la clasificación práctica de los enfermos según sus signos y síntomas (flujo de atención de los pacientes), el reordenamiento de los servicios y el aseguramiento mínimo de algunos recursos.

\section{LA CAPACITACIÓN DEL PERSONAL MÉDICO Y DE ENFERMERÍA}

La actividad de capacitación constituye una inversión y como tal se debe programar, cuantificar, controlar y evaluar. El tiempo y los recursos que se utilizan en la capacitación pueden equivaler a los gastos en vacunas y medicamentos de otras enfermedades y que no están disponibles para el dengue.

El adiestramiento estará dirigido a todo el personal médico y de enfermería (52) que trabaja en los hospitales y los centros de atención primaria y debe perseguir los siguientes objetivos:
- Mejorar el diagnóstico clínico mediante el conocimiento de las manifestaciones clínicas y humorales de la enfermedad, así como la secuencia en que se presentan. Con esto se logra mejorar el diagnóstico preliminar de la enfermedad sin desatender el diagnóstico diferencial.

- Aplicar una adecuada clasificación clínica de los pacientes, que sea sencilla y dinámica, de manera que se pueda aplicar en cualquier lugar y facilite la introducción de cambios rápidos en el tratamiento.

- Mejorar el tratamiento mediante el establecimiento de conductas acordes con cada etapa clínica de la enfermedad, atender las complicaciones precozmente y evitar el sobretratamiento y la indicación de medicamentos innecesarios.

Es sumamente importante saber identificar correctamente la secuencia de las manifestaciones clínicas y de los resultados de laboratorio para poder diferenciar el dengue de otros trastornos o enfermedades que pudieran producir alteraciones parecidas con distinto orden de presentación (leptospirosis, infección meningocócica, influenza, septicemia y otras). Además, esta es la única posibilidad de detectar precozmente a los pacientes con dengue en peligro de evolucionar hacia la forma clínica grave de DH/SCD.

Generalmente, la primera manifestación clínica es la fiebre, de intensidad variable, aunque puede ser antecedida por diversos síntomas. La fiebre generalmente se acompaña de cefalea y vómitos, así como de dolores en el cuerpo, que constituye el cuadro del llamado dengue clásico. En los niños es frecuente que la fiebre sea la única manifestación clínica, aunque a veces se acompaña de trastornos digestivos. La fiebre puede durar de 2 a 7 días y puede haber trastornos bastante característicos del gusto y enrojecimiento de la faringe, aunque otros síntomas y signos del aparato respiratorio no son frecuentes ni importantes. Pueden presentarse dolor abdominal discreto y diarrea (53), esto último más frecuentemente en los pacientes menores de dos años (54) y en los adultos (55). En los primeros días aparece exantema en un porcentaje variable de los pacientes; no se ha demostrado que el exantema sea un factor de mejor o peor pronóstico.

Estas manifestaciones se mantienen al menos durante las primeras 48 horas de la enfermedad y pueden extenderse durante algunos días más. En esta etapa febril de la enfermedad no es posible predecir si el paciente tendrá síntomas y signos de dengue clásico todo el tiempo hasta curarse de forma espontánea o si evolucionará a DH/SCD.

Entre el tercer y el sexto día después de la aparición de los primeros síntomas en los niños y entre el cuarto y el sexto día en los adultos (como 
FIGURA 1. Etapas clínicas del dengue hemorrágico

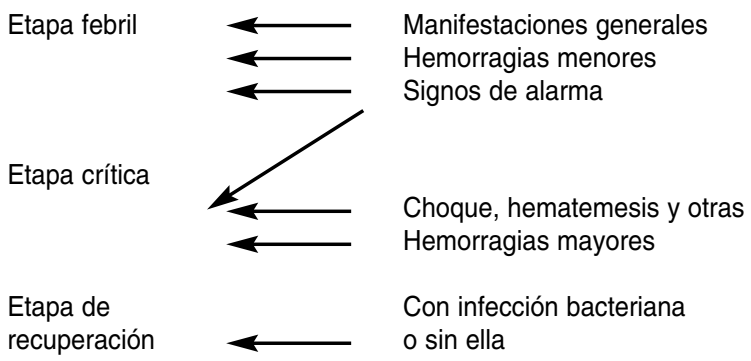

período más frecuente, pero no exclusivo de los enfermos que evolucionan hacia DH/SCD), la fiebre desciende, el dolor abdominal se hace intenso y constante, se constata la presencia de derrame pleural o ascítico, los vómitos aumentan en frecuencia y comienza la etapa crítica de la enfermedad. Este es el momento en que con mayor frecuencia se presenta el choque y cuando se hace evidente la hepatomegalia. La presencia de signos de alarma anuncia esta etapa (figura 1).

El hematócrito es normal al principio y va ascendiendo a la vez que los estudios radiológicos de tórax o la ultrasonografía abdominal muestran ascitis o derrame pleural derecho o bilateral. La máxima elevación del hematócrito coincide con el choque. Lo más llamativo es que el recuento plaquetario muestra un descenso progresivo hasta llegar a las cifras más bajas durante el día del choque para después ascender rápidamente y normalizarse en pocos días. El choque se presenta cuatro o cinco veces más frecuentemente cuando baja la fiebre o en las primeras 24 horas después de su desaparición que durante la etapa febril (20).

No todos los signos clínicos de choque tienen que estar presentes desde el inicio. Basta constatar el estrechamiento de la tensión arterial diferencial o de la presión del pulso hasta una diferencia de $20 \mathrm{~mm} \mathrm{Hg}$ o menos entre la tensión arterial sistólica y la diastólica. Esto generalmente está precedido por o acompañado de signos de inestabilidad hemodinámica (taquicardia, frialdad y el enlentecimiento del llenado capilar, entre otros). Por tanto, no es necesario esperar hasta que se produzca la hipotensión para diagnosticar el choque.

La mayoría de las veces, los signos de choque duran varias horas. Cuando el choque es prolongado o recurrente, o sea, que dura más de 12 ó 24 horas o, excepcionalmente, más de 48 horas, se ven edemas intersticiales en las imágenes radiológicas de los pulmones, semejantes a veces a las lesiones neumónicas. Más adelante puede instalarse un sín- drome de dificultad respiratoria por edema pulmonar no cardiógeno, lo que hace el pronóstico más reservado aun.

Después de la etapa crítica, el enfermo pasa un tiempo variable en la etapa de recuperación, que también requiere la atención del equipo de APS, pues durante este período se debe eliminar fisiológicamente el líquido extravasado hasta normalizarse todas las funciones vitales. En niños y adultos sanos, esta diuresis aumentada se tolera bien, pero se debe vigilar este proceso en personas con cardiopatías, nefropatías y ancianos. Se debe vigilar también la aparición de una posible coinfección bacteriana, casi siempre pulmonar, así como del llamado exantema tardío (10 días o más después del descenso de la fiebre). Algunos pacientes adultos se mantienen muchos días con astenia y otros refieren bradipsiquia durante semanas.

\section{EXÁMENES DE LABORATORIO Y DIAGNÓSTICO POR IMÁGENES}

Se debe resaltar que durante una epidemia, el laboratorio clínico no debe sobrecargarse con investigaciones que no sean estrictamente las necesarias, pues esto puede menoscabar la calidad de los resultados.

Es probable que el médico que atiende a un paciente con dengue pida un recuento leucocitario en busca de leucopenia, cuya fórmula diferencial hará evidente la neutropenia propia de la fase inicial de la enfermedad, algunas células en banda y linfocitos atípicos. El hematócrito y el recuento plaquetario serán los exámenes de laboratorio clínico indispensables para el diagnóstico del DH/SCD, aunque no son estrictamente necesarios durante el seguimiento de los casos febriles sospechados de tener dengue si no hay sangrados espontáneos o un resultado positivo a la prueba del torniquete. Los enfermos que requieren análisis de hematócrito y recuento plaquetario generalmente los necesitan seriados durante varios días.

Se ha asociado el recuento leucocitario $>6000$ células $/ \mathrm{mm}^{3}$ con la progresión del enfermo hacia DH/SCD, al menos en adultos (56). En Río de Janeiro, los resultados de laboratorio durante la epidemia de 2002 demostraron la importancia de la leucocitosis y la hemoconcentración como indicadores pronósticos de la evolución a formas graves de la enfermedad debido a la frecuencia de estas alteraciones en los enfermos que luego fallecieron. Lo mismo ocurrió con la elevación de los valores de las transaminasas hepáticas, principalmente de la glutámico-oxalacética (TGO) (50).

El estudio del paciente debe completarse según el cuadro clínico, las posibilidades de la ins- 
CUADRO 2. Alteraciones diagnosticables por ultrasonografía abdominal en casos de dengue hemorrágico

- Derrame pleural
- Ascitis
- Engrosamiento de la pared de la vesícula biliar y presencia de
líquido perivesicular
- Hepatomegalia
- Pancreatomegalia
- Acumulación de líquido en las zonas pararrenal y perirrenal

titución y el tipo de atención que se preste: ambulatoria o con hospitalización. En el caso de los pacientes hospitalizados puede requerirse la realización de un coagulograma completo y la evaluación del tiempo de sedimentación eritrocitaria, la evaluación de la concentración de proteínas totales, ionograma y gasometría, así como análisis de nitrógeno de urea, creatinina, transaminasas hepáticas u otras enzimas en sangre que expresen la citólisis hepática. De ser necesario se debe prescribir un medulograma.

En determinados casos, el médico puede requerir un diagnóstico diferencial, para lo cual es necesario hacer un hemocultivo, la prueba de la gota gruesa, un estudio del líquido cefalorraquídeo (citoquímico y bacteriológico) y otras pruebas más específicas. Los estudios radiológicos de tórax y la ultrasonografía abdominal son muy útiles en el diagnóstico del dengue hemorrágico (cuadro 2), al igual que los electrocardiogramas y los ecocardiogramas si se cree que hay afectación miocárdica (57).

Es importante destacar que el estudio serológico más utilizado para el diagnóstico de dengue, la detección de anticuerpos específicos de tipo IgM, debe indicarse a partir del quinto o, preferentemente, del sexto día. Por tanto, esta prueba no ayuda al médico de asistencia a decidir la conducta terapéutica, pues el paciente se puede agravar a partir del tercer o cuarto día. No obstante, es importante indicar este estudio serológico, que completa el trípode diagnóstico junto con la clínica y la epidemiología. Otro estudio serológico importante consiste en la determinación de anticuerpos IgG específicos contra el dengue que deben indicarse a partir de la tercera semana del comienzo de la enfermedad. Las pruebas de laboratorio para identificar antígenos virales, en particular para identificar algunas de las proteínas no estructurales del virus del dengue, están en proceso de validación e introducción en la práctica $(58,59)$. El diagnóstico molecular mediante la reacción en cadena de la polime- rasa y el cultivo para el aislamiento y la identificación del virus son de utilidad, aunque por su costo y complejidad no están disponibles en la mayoría de las instalaciones sanitarias. Para su realización es necesario establecer coordinaciones con los laboratorios que tengan capacidad para realizar estos análisis.

\section{LA EDUCACIÓN DE LA POBLACIÓN}

Cuando en una ciudad o región hay transmisión de dengue, la población debe estar informada no solamente de las acciones que debe realizar para controlar el vector, sino también de los síntomas y signos de esta enfermedad (60).

Todas las actividades educativas deben tener como objetivo que las familias participen en su autocuidado, soliciten a tiempo los servicios médicos, eviten la automedicación, reconozcan precozmente los sangrados cutáneos (petequias), estén apercibidos de que el día que baja la fiebre es el de mayor riesgo de presentar complicaciones (y durante las 48 horas siguientes) y estén vigilantes de los síntomas y signos de alarma, en particular del dolor abdominal intenso y mantenido y de los vómitos frecuentes (61). Debe, además, hacerse hincapié en la rehidratación oral desde el primer día de la enfermedad (62).

El nivel de información y la mejor forma de hacerle comprender y aceptar el mensaje a la población dependerán en cada caso del nivel cultural del grupo poblacional en cuestión, sus conocimientos previos sobre el dengue y su acceso a los servicios de salud, entre otros factores. Por tanto, el diseño y la ejecución de las actividades educativas deberán hacerse en conjunto entre técnicos de la salud y profesionales de la comunicación social.

Las acciones de educación para la salud deben ser de tipo informativo y los medios masivos (prensa escrita, radio, televisión, vallas anunciadoras y otros) pueden cumplir una excelente función si se recibe la orientación adecuada. Se debe prestar atención particular a los periodistas, directores de programación, editores y firmas anunciadoras, mediante la organización de talleres y otras modalidades presenciales que garanticen su participación constructiva en este empeño educativo, pero cuidando de no crear pánico en la población. No se trata solamente de informar, sino de cambiar actitudes.

Los maestros y profesores de los distintos niveles de enseñanza se deben convertir en voceros indispensables, sobre todo si son capaces de multiplicar sus esfuerzos mediante la incorporación de sus estudiantes. Esa educación "en cascada" puede llegar a cada familia en su propio hogar. Se debe incorporar 
a los líderes formales e informales de la comunidad a estas actividades de educación de la población.

Durante las epidemias de dengue, los estudiantes de medicina y enfermería y los activistas sanitarios de otros sectores pueden organizarse para visitar los hogares en la doble función de búsqueda activa de casos y de educación. Esta acción ha demostrado ser factible, económica y eficiente y se debe ejecutar de forma coordinada con el equipo de APS y con las instancias que puedan aportar materiales impresos amenos y creativos para distribuir durante esas visitas.

Es muy importante lograr que los médicos, enfermeros y demás técnicos de la salud incorporen la función educativa a su quehacer cotidiano. La práctica de la asistencia sanitaria se completa con las acciones de promoción y prevención, que deben ejecutarse como parte del acto médico, dentro y fuera de los consultorios, en un lenguaje comprensible y con el respaldo que brindan la autoridad y el prestigio que la población reconoce habitualmente en estos profesionales.

\section{LA CLASIFICACIÓN PRÁCTICA DE LOS ENFERMOS: CONDUCTA A SEGUIR Y TRATAMIENTO}

No hay dudas acerca de la conveniencia e importancia de disponer de un protocolo de tratamiento para los enfermos con dengue $(63,64)$. Los médicos necesitan un método simplificado para clasificar a los pacientes durante una epidemia. Se trata de una clasificación para la acción, es decir, para decidir las conductas respecto de adónde remitir al paciente, qué exámenes de laboratorio indicarle, cómo tratarlo y cuáles signos de alarma vigilar (24).

El siguiente método es aplicable en todos los niveles de atención sanitaria, ya sean unidades de atención primaria, servicios de urgencia o salas de hospitalización, así como en las visitas a los hogares. En estas visitas, los médicos, enfermeros o estudiantes adiestrados para esta tarea deben realizar pesquisas activas siempre que sea posible.

Se debe interrogar a todos los pacientes con fiebre, tanto desde el punto de vista clínico como epidemiológico, y precisar el inicio y la duración de los síntomas. Además, se debe hacer el examen físico de los pacientes para diagnosticar otras posibles causas de fiebre que concurren durante las epidemias de dengue. Cada vez que un médico, enfermero o estudiante adiestrado atienda un caso sospechado de dengue, deberá tratar de responder a las siguientes preguntas:

¿Tiene dengue?

¿Tiene sangrado?
¿Presenta algún signo de alarma?

¿Está en choque?

Según los síntomas y signos que se observen en un momento determinado de la enfermedad y las respuestas a esas preguntas básicas, se puede clasificar al paciente como:

A. Caso febril

B. Caso con fiebre y petequias (u otro sangramiento)

C. Caso con signos de alarma

D. Caso con signos de choque

Esta clasificación determinará las decisiones clínicas, de laboratorio, de hospitalización y terapéuticas que deberán tomarse con el paciente. La valoración de cada caso debe ser dinámica, pues el enfermo puede presentar cambios en su cuadro clínico que lo hagan pasar de un grupo a otro en corto tiempo, de acuerdo con el flujo de atención a los pacientes con dengue.

Todos los pacientes que presenten un cuadro clínico sugestivo de dengue se deben incorporar al grupo A. Si no presentan signos de alarma ni sangrados espontáneos (y la prueba del torniquete resulta negativa), se le debe indicar un tratamiento ambulatorio de tipo sintomático (sin aspirina o medicamentos antiinflamatorios no esteroideos) y se considerará como un caso sospechado de dengue hasta que - a partir del sexto día de fiebre - se le tome una muestra de sangre para determinar la presencia de anticuerpos IgM específicos contra el dengue. Se le debe brindar toda la información necesaria sobre la enfermedad y los signos de alarma que anuncian el peligro de complicaciones y se le debe mantener en observación, especialmente a partir de la caída de la fiebre. Se le debe indicar ingerir abundante cantidad de líquidos durante toda la etapa febril, ya que esta medida ha contribuido a disminuir el número de hospitalizaciones por dengue (65).

Si el paciente ha tenido algún sangrado espontáneo (petequias, epistaxis u otro) o la prueba del torniquete resulta positiva, pasa al grupo $\mathrm{B}$ y, aunque puede tratarse de un caso de dengue clásico con sangrado, en la práctica debe considerarse como un posible caso de dengue hemorrágico. Se le debe indicar un recuento de plaquetas y hematócrito (que serán repetidos con la frecuencia adecuada para el caso, pero al menos una vez al día) y preferentemente se le debe hospitalizar para lograr un seguimiento más constante (cuadro 3). Los recuentos plaquetarios que muestren una disminución progresiva, así como el aumento del hematócrito, serán también criterios de hospitalización.

No es necesario realizar la prueba del torniquete cuando ya existen sangrados espontáneos. 
CUADRO 3. Criterios clínicos de hospitalización durante una epidemia de dengue

- Dolor abdominal intenso y sostenido
- Vómitos numerosos
- Irritabilidad, somnolencia o ambos
- Manifestaciones hemorrágicas, independientemente de la cifra
de plaquetas
- Hemoconcentración, ascitis o derrame torácico
- Sudoración profusa, lipotimia o gran debilidad (postración)
durante la defervescencia
- Hipotensión arterial, frialdad de los miembros
- Dolor torácico, dificultad respiratoria, disminución del murmullo
vesicular o cualquier signo que indique alteraciones de la
ventilación
- Cianosis

Esta prueba se realiza como tamizaje para identificar precozmente al enfermo de dengue que probablemente va a sangrar y así poder decidir su hospitalización temprana $\mathrm{u}$ observación prioritaria mediante consultas repetidas y recuentos plaquetarios seriados. Para la interpretación de la prueba del torniquete debe tenerse en cuenta que un resultado positivo debe conducir a una observación cercana del caso y que el resultado negativo no permite descartar una evolución al DH/SCD (66).

Se debe mantener la vigilancia clínica de todos los pacientes hospitalizados por dengue y se deben anotar sus signos vitales (tensión arterial, frecuencia respiratoria y frecuencia cardíaca), el ritmo diurético y el equilibrio de líquidos, así como la aparición de dolor abdominal intenso u otro signo de alarma. Esta vigilancia debe mantenerse durante toda la etapa crítica hasta la etapa de recuperación.

La ausencia de sangrados espontáneos y la negatividad a la prueba del torniquete no significan que el enfermo no pueda evolucionar hacia formas graves de la enfermedad. En ese sentido, un colectivo carioca que describió su experiencia durante la epidemia de 2002 con el serotipo 3 apuntó que: “... algunas veces, los profesionales de la salud y los propios pacientes valoraron de manera especial la presencia de manifestaciones hemorrágicas y los resultados del recuento plaquetario. No obstante, destacamos que la principal característica fisiopatológica asociada con las formas graves de $\mathrm{DH} / \mathrm{SCD}$ fue la extravasación de plasma evidenciada por la hemoconcentración y por los signos de alarma" (36).

Si el paciente se presenta con signos de alarma o los desarrolla durante su hospitalización, se debe clasificar dentro del grupo C, pues el médico debe estar preparado para prevenir el choque. Lo mismo sucede si muestra una elevación progresiva del hematócrito o si el hematócrito ya está elevado en $20 \%$ o más, lo cual es un indicador de la hemoconcentración provocada por una extravasación clínicamente importante de plasma.

El dolor abdominal intenso y sostenido $\mathrm{u}$ otro signo de alarma indica el comienzo del deterioro clínico del enfermo con dengue $(67,68)$. Ese es el momento de comenzar la rehidratación enérgica por vía intravenosa y de brindarle cuidados progresivos para salvarle la vida.

En Río de Janeiro durante la epidemia de 2002, 53,8\% de los pacientes que fallecieron (todos adultos) tuvieron dolor abdominal intenso y sostenido y $41 \%$ presentaron vómitos incoercibles (50). En Bucaramanga, Colombia, el estudio de pacientes pediátricos atendidos durante 10 años demostró que los principales signos de alarma en la gran mayoría de los niños con dengue que sufrieron choque fueron el dolor abdominal intenso, a veces asociado con vómitos frecuentes, hipotermia o somnolencia (69).

Si ya están presentes los signos de choque y la tensión arterial diferencial está próxima a $20 \mathrm{~mm} \mathrm{Hg}$ o hay una franca hipotensión, se considerará al paciente dentro del grupo $\mathrm{D}$ y se le deberá administrar el tratamiento indicado para el choque hipovolémico, que consiste en rehidratación enérgica con soluciones cristaloides por vía intravenosa (70), entre otras medidas $(71,72)$. La administración de albúmina humana, gelatinas y otros coloides no son el tratamiento de elección y solamente se deben utilizar en cantidades mínimas para recuperar la tensión arterial en los casos en que esto no se pudo lograr por otros medios. Una vez recuperada la tensión arterial, se debe continuar la rehidratación con soluciones cristaloides (73).

Este tratamiento es prácticamente el mismo que se aplica a los pacientes con signos de choque por deshidratación en el curso de una enfermedad diarreica aguda (10). El aporte enérgico de líquidos debe iniciarse tan pronto se observen los signos de choque y en el lugar donde se encuentre el paciente, incluso un servicio de APS, aunque después se traslade al hospital.

En el tratamiento del síndrome de choque por dengue no se utilizan corticoides ni heparinas. Las drogas inotrópicas (dopamina, dobutamina) deberán indicarse solamente cuando esté normalizada la volemia o en los enfermos que tras la reposición adecuada del volumen de líquido extravasado no mejoren la perfusión (73). Se ha encontrado que la hematemesis o la melena durante el choque son factores asociados con la mortalidad (74).

Después de pasada la gravedad y cuando se está preparando el egreso del paciente con $\mathrm{DH} /$ 
CUADRO 4. Criterios de egreso de pacientes hospitalizados con dengue

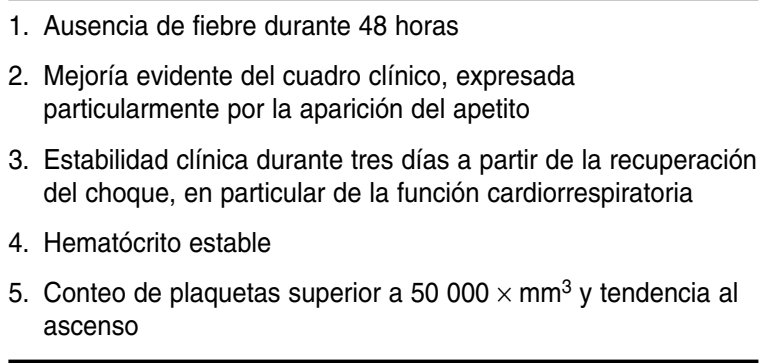

SCD (cuadro 4), el médico estará en condiciones de clasificarlo según los criterios de gravedad de la enfermedad (I-IV) establecidos por la OMS. (75)

Si bien el dengue puede ser mortal, hay consenso en que el pronóstico de la enfermedad es bueno si el tratamiento de sostén y de rehidratación se inicia a tiempo y de manera adecuada (76).

\section{EL REORDENAMIENTO DE LOS SERVICIOS Y EL ASEGURAMIENTO MÍNIMO DE RECURSOS}

Como se ha visto en el transcurso del trabajo, durante una epidemia es necesario reordenar el sistema de atención, diagnóstico y tratamiento de los pacientes con dengue. A continuación se exponen las principales pautas para cumplir ese objetivo.

- Reordenar los servicios de urgencia de los hospitales con un enfoque dirigido prioritariamente a los enfermos con sospecha de dengue y reforzar su personal con un número suficiente de profesionales adiestrados que permita satisfacer la elevada demanda de servicios que impone una epidemia. Lo mismo se aplica a los servicios de APS, donde se recomienda crear unidades de dengue con personal facultativo las 24 horas del día.

- Aplicar el esquema de clasificación y atención a enfermos con dengue recomendado por la OPS (24) y adecuado al lugar. Facilitar su cumplimiento mediante la colocación de carteles con ese esquema de atención a los pacientes en los locales de consulta.

- Realizar los exámenes de laboratorio estrictamente indispensables, según lo normado al efecto, para garantizar la calidad y confiabilidad de los mismos.

- Utilizar criterios de hospitalización bien definidos y garantizar camas para todos los pacientes que la requieran. Pueden crearse instalaciones provisionales en centros de APS para brindar atención inmediata y transitoria (hasta su tras- lado al hospital) a los enfermos que requieran rehidratación por vía parenteral.

- Organizar una adecuada observación clínica de cada paciente hospitalizado. Se pueden utilizar para ello alumnos de medicina o de enfermería, previamente adiestrados en los aspectos elementales que deben vigilar de cerca a fin de identificar signos precoces de agravamiento. Convertir en salas de observación y tratamiento de casos de dengue alguna(s) de las salas o unidades de cuidados convencionales.

- Brindar atención especial a los pacientes más graves: agrupar al personal más experimentado y concentrar los equipos disponibles para utilizarlos con el máximo aprovechamiento en salas de cuidados especiales para el tratamiento inicial de las complicaciones y en unidades de cuidados intensivos.

- Crear una comisión técnica multidisciplinaria de alto nivel científico que emita las orientaciones metodológicas y evalúe la marcha y los resultados del plan. Esto permite aplicar un procedimiento unificado que garantice la calidad de la asistencia médica.

- Garantizar la disponibilidad de analgésicos, antipiréticos y frascos de soluciones de rehidratación parenteral (cristaloides) y sustancias coloides (plasma, albúmina), así como los reactivos que satisfagan la demanda de un incremento de recuentos plaquetarios y estudios radiológicos de tórax. En la práctica, los equipos de ultrasonografía han demostrado que pueden servir para muchas de las indicaciones de estudios radiológicos.

\section{CONCLUSIONES}

El tratamiento exitoso de las formas graves de dengue se apoya en una estrategia cuya esencia es preventiva: a) el seguimiento de todos los casos febriles sospechados de dengue y la aplicación de medidas para prevenir el desequilibrio hidromineral mediante el aporte de líquidos abundantes por vía oral; b) la identificación temprana de los signos de alarma para iniciar precozmente la rehidratación parenteral que permita prevenir el choque; y c) el tratamiento enérgico del choque con soluciones electrolíticas en cantidad suficiente para prevenir complicaciones - tales como la coagulación intravascular diseminada, los sangrados abundantes y el síndrome de hipoperfusión-reperfusión que conduce al fallo de múltiples órganos-, y evitar la sobrehidratación con el objetivo de prevenir el edema pulmonar causante del síndrome de dificultad respiratoria.

Los métodos de atención se desarrollan y acercan cada vez más a los escenarios de la APS y a la comunidad, con la incorporación progresiva de enfermeros, técnicos de la salud y estudiantes, así 
como de los promotores que acompañan al personal sanitario en la búsqueda activa de casos.

Es necesario mantener y aumentar la capacidad resolutiva del hospital durante las epidemias y de las figuras del médico y el enfermero como principales protagonistas en sus distintos espacios, desde el servicio de urgencias hasta la unidad de cuidados intensivos. Esto es aplicable a los hospitales públicos, a las clínicas privadas y a las demás instituciones de salud, como las dependientes del seguro social.

La calidad de la atención médica determina en gran medida la ausencia o poca frecuencia de mortalidad por dengue y depende a su vez de un conjunto de medidas organizativas y de capacitación que se deben diseñar y adecuar a cada lugar. Estas medidas se deben poner en práctica como parte de las acciones de prevención y control antes de que surja la epidemia.

La preparación de los recursos humanos es la medida más valiosa e importante. Esta abarca de modo prioritario el trabajo informativo-educativo a la población hasta lograr que participe activamente en su autocuidado y pueda reconocer oportunamente los signos que anuncian la gravedad. Todos los recursos se deben reordenar también en función de la prioridad que reclama una epidemia de dengue y se deben controlar debidamente.

\section{SYNOPSIS \\ Preventing deaths from dengue: a space and challenge for primary health care}

Dengue is an arthropod-borne viral disease whose frequency has increased steadily in the Americas over the past 25 years. The type of dengue that carries the highest mortality is the clinical variant known as dengue hemorrhagic fever/dengue shock syndrome (DHF/DSS). Even though no vaccine or drug against the disease is available, successful management consists of preventing serious illness through patient followup and monitoring danger signals so as to be able to initiate aggressive intravenous rehydration and prevent shock or treat it early and successfully. These measures are also useful in preventing other complications, such as massive hemorrhage, disseminated intravascular coagulation, multiple organ failure, and respiratory failure due to non-cardiogenic pulmonary edema. Primary health care (PHC) settings and the community are ideal spaces for this type of preventive management based on health education and active case detection. It involves training all medical and nursing staff, students, and community health workers, as well as reorganizing health care in PHC units and hospitals and redistributing available resources during a dengue epidemic.

Key words: dengue, dengue hemorrhagic fever, primary health care, prevention, mortality.

\section{REFERENCIAS}

1. Guzmán M, Kourí G, Díaz M, Llop A Vázquez S, González D, et al. Dengue, one of the great emerging health challenges of the 21st century. Expert Rev Vaccines. 2004;3(5):511-20.

2. Gubler D. The emergence of epidemic dengue fever and dengue hemorrhagic fever in the Americas: a case of failed public health policy. Rev Panam Salud Publica. 2005;17(4):221-4.

3. Calisher $\mathrm{CH}$. Persistent emergence of dengue. Emerg Infect Dis. 2005;11(5): 738-9.

4. Martínez E. Dengue. En: GonzálezSaldaña N, Torales Torales AN, Gómez Barreto D, eds. Infectología clínica pediátrica. México, D.F.: Editorial Trillas; 1997. Pp. 589-95.

5. Bosco Siquiera J, Turchi Martelli CM, Evelim Coelho G, da Rocha Simplicio AC, Hatch DL. Dengue and dengue hemorrhagic fever, Brazil, 1981-2002. Emerg Infect Dis. 2005;11(1):48-53.

6. Teichmann D, Gobels K, Neidrig M, Grobusch MP. Dengue virus infection in travellers returning to Berlin, Germany: clinical, laboratory and diagnostic aspects. Acta Trop. 2004;90(1):87-95.

7. Peragallo MS, Nicoletti L, Lista F, D'Amelio R. Probable dengue virus infection among Italian troops, East Timor, 19992000. Emerg Infect Dis. 2003;9(7):876-80.

8. Mc Bride WJH. Deaths associated with dengue hemorrhagic fever: the first in Australia in over a century. Med J Australia. 2005;183(1):35-7.

9. Buhl MR. Flavivirus infections; yellow fever, dengue fever and Japanese encephalitis. Ugeskr Laeger. 2005;167(43): 4076-80.

10. Halstead SB. Viral hemorrhagic fevers. In: Burg FD, Ingelfinger JR, Wald ER, Polin RA, eds. Gellis and Kagan's Current Pediatric Therapy 16. Philadelphia: WB Saunders Co; 1999. Pp. 108-11.

11. Schexneider KI, Reedy EA. Thrombocytopenia in dengue fever. Curr Hematol Rep. 2005;4(2):145-8.

12. Gomber S, Ramachandran VG, Kumar S, Agarwal KN, Gupta P, Dewan DK. Hematological observations as diagnostic markers in dengue hemorrhagic fever-a reappraisal. Indian Pediatrics. 38(5):477-81.

13. Srichaikul T, Nimmannitya S. Haematology in dengue and dengue hemorrhagic fever. Baillieres Best Pract Res Clin Haematol. 2000;13(2):261-76.

14. Nimmannitya S. Dengue and dengue hemorrhagic fever: pearls and pitfalls in diagnosis and management. In: Kalayanarooj S (ed). Studies/collaborative studies on dengue infections/dengue hemorrhagic fever at Queen Sirikit National Institute of Child Health (Children's Hospital). Bangkok: WHO Collaborating Centre for Case Management of Dengue/DHF/DSS, Queen Sirikit National Institute of Child Health; 2003. Pp. 324-31.

15. Lum LC, Abdel-Latif MA, Goh AY, Chan PW, Lam SK. Preventive transfusion in dengue shock syndrome-is it necessary? J Pediat. 2003;143(5):682-4.

16. Upadhyay M, Singhi S, Murlidharan J, Kaur N, Majumdar S. Randomized evaluation of fluid resuscitation with crystalloid (saline) and colloid (polymer 
from degraded gelatine in saline) in pediatric septic shock. Indian Pediatr. 2005;42(3):223-31.

17. World Health Organization. Primary health care. In: Dengue hemorrhagic fever: diagnosis, treatment, prevention and control. 2nd ed. Geneva: WHO; 1997. Pp. 67-9.

18. Montenegro D, Lacerda HR, Lira TM, Oliveira DS, Lima AA, Guimaraes MJ, et al. Clinical and epidemiological aspects of the dengue epidemic in Recife, PE, 2002. Rev Soc Bras Med Trop. 2006; 39(1):9-13.

19. Martínez E. Dengue. Rio de Janeiro: Fiocruz; 2005.

20. Martínez E. Dengue y dengue hemorrágico: aspectos clínicos. Salud Publica Mex. 1995;37(Suppl):29-44.

21. Rigau-Pérez JG, Clark GC, Gubler DF, Reiter P, Sanders EJ, Vordam V. Dengue and dengue haemorrhagic fever. Lancet. 1998;352:971-7.

22. World Health Organization, Department of Child and Adolescent Health and Development. Dengue, dengue hemorrhagic fever and dengue shock syndrome in the context of the integrated management of childhood illness. Geneva: WHO; 2005. (WHO/ $\mathrm{FCH} / \mathrm{CAH} / 05.13)$

23. World Health Organization. Guidelines for treatment of dengue fever/dengue hemorrhagic fever in small hospitals. New Delhi: WHO Regional Office for South-East Asia; 1999.

24. Organización Panamericana de la Salud. Dengue y dengue hemorrágico. Guías para su prevención y control. Washington, D.C.: OPS; 1995. (Publicación Científica No. 548)

25. World Health Organization. Dengue haemorrhagic fever: diagnosis, treatment, prevention and control. Management of clinical care. Organizational aspects. Geneva: WHO; 1997.

26. República del Perú, Ministerio de Salud, Oficina General de Epidemiología. Normas para la prevención y control del dengue. Lima: Ministerio de Salud de Perú; 1990.

27. República Federativa de Brasil, Ministerio de Salud, Fundación Nacional de Salud. Programa nacional de control de Dengue. Brasilia: Ministerio de Salud; 2002.

28. República de Venezuela, Ministerio de Sanidad y Asistencia Social. Guía para el diagnóstico, tratamiento, vigilancia epidemiológica, prevención y control del dengue. Caracas: Ministerio de Sanidad y Asistencia Social; 1990.

29. Pizarro D, Vargas W, Terwes G, Vives JE, Solano A, Martínez E, et al. Guías para el diagnóstico y tratamiento del dengue y dengue hemorrágico. San José: Ministerio de Salud de Costa Rica, Caja Costarricense de Seguro Social, Organización Panamericana de la Salud 1993.

30. Pereira R, Rubio J, Madariaga A, Gil E, Pineda C, Chiesa M. Lineamientos para el manejo intrahospitalario del paciente con dengue. Tegucigalpa: Secretaría de Salud de Honduras, Organización Panamericana de la Salud; 1994

31. Luna JA, Amador R, Martínez E. Atención del paciente con dengue. Santa Fe de Bogotá: Ministerio de Salud de la República de Colombia; 1990.

32. Villalobos de Chacón I, Miño León G Real Cotto J, Andrade Maingon A. Aspectos prácticos del manejo clínico, diagnóstico y tratamiento del dengue/ dengue hemorrágico. Guayaquil, Ecuador: Ministerio de Salud Pública de Ecuador, Subsecretaría Nacional de Medicina Tropical; 2001.

33. República Dominicana, Secretaría de Estado de Salud Pública y Asistencia Social, Comisión Nacional para la Prevención y Control del Dengue. Dengue y dengue hemorrágico. Diagnóstico y tratamiento. Santo Domingo, República Dominicana: Secretaría de Estado de Salud Pública y Asistencia Social, Organización Panamericana de la Salud; 2005.

34. Rigau-Pérez JG, Clark GG. Cómo responder a una epidemia de dengue: visión global y experiencia en Puerto Rico. Rev Panam Salud Publica. 2005; 17(4):282-93.

35. Oliveira Zagne SM, Leite Muñiz MG. Integrando conceitos à práctica diária na conduta ao paciente com dengue. Saúde em Foco/Informe Epidemiol Saúde Colectiva (Rio de Janeiro). 2002;24: $115-21$.

36. Wakimoto MD, Azevedo MB, Sant' Anna de Oliveira J, Vommaro RV, Dornas JE. A experiência de um hospital público na vigilância e assistência aos casos de dengue durante a epidemia de 2002. Saúde em Foco/Informe Epidemiol Saúde Colectiva (Rio de Janeiro). 2002;24:25-62.

37. Martínez Torres E. Dengue y dengue hemorrágico. Buenos Aires: Universidad Nacional de Quilmes; 1998.

38. Kourí G, Guzmán MG, Bravo J. Hemorrhagic dengue in Cuba: history of an epidemic. PAHO Bull. 1986;20:24-30

39. Rojas Ochoa F. Modelo de lucha antiepidémica. Rev Cubana Salud Publica. 2003;29(1):7

40. Guzmán MG, Triana C, Bravo J, Kourí G. Estimación de las afectaciones económicas como consecuencia de la epidemia de dengue hemorrágico ocurrida en Cuba en 1981. Rev Cubana Med Trop. 1992;44(1):13-7.

41. Dotres Martínez C. Principales aspectos organizativos de la atención médica durante la epidemia de dengue hemorrágico en Cuba. En: Martínez E, Dotres C, Guzmán MG, Kourí G, eds. Dengue hemorrágico en el niño. Santo Domingo, República Dominicana: Editora Universitaria; 1985. Pp. 181-92.

42. Zamora Ubieta F. Atención de adultos durante la epidemia de dengue hemorrágico en Cuba, 1981. Clasificaciones utilizadas. La Habana: Ministerio de Salud Pública; 1982.
43. Valdés L, Guzmán MG, Kourí G, Delgado J, Carbonell I, Cabrera MV, et al. La epidemiología del dengue hemorrágico en Santiago de Cuba, 1997. Rev Panam Salud Publica. 1999;(6)1: 16-24.

44. De la Torre Montejo E, López Pardo C, Márquez M, Gutiérrez Muñiz JA, Rojas Ochoa F. Salud para todos sí es posible. La Habana: Soc Cubana de Salud Pública; 2004

45. González D, Castro OE, Kourí G, Pérez J, Martínez E, Vázquez S, et al. Classical dengue hemorrhagic fever resulting from two dengue infections spaced 20 years or more apart: Havana dengue 3 epidemic, 2001-2002. Int J Infect Dis. 2005;9(5):280-5.

46. Lemus Lago ER, Estévez Torres G, Velázquez Acosta JC. Campaña por la esperanza. La lucha contra el dengue. La Habana: Editora Política; 2002.

47. Méndez A, González G. Manifestaciones clínicas inusuales del dengue hemorrágico en niños. Biomédica. 2006;26: 61-70.

48. Bandyopadhyay S, Lum LCS, Kroeger A. Classifying dengue: a review of the difficulties in using the WHO case classification for dengue hemorrhagic fever. Trop Med Internat Health. 2006;2(8): 1238-55.

49. Teixeira MG, Lima Barreto M, Costa MCN. Dengue no Brasil: as epidemias de 1981 a 2002. Saúde em Foco/Informe Epidemiol Saúde Colectiva (Rio de Janeiro). 2002;24:35-46.

50. Azevedo MB, Kneipp MB, Baran M, Araújo Nicolai CC, Caldas D, Fernández SR, et al. O previsível e o prevenivel: mortes por dengue na epidemia carioca. Saúde em Foco/Informe Epidemiol Saúde Colectiva (Rio de Janeiro). 2002; 24:65-80.

51. Kalayanarooj S, Chansiriwongs V, Vatcharasaevee V, Waleerattanapa $R$, Nimmannitya S. Capacity building for case management of dengue hemorrhagic fever. Thai Pediatr J. 2000;7: 178-9.

52. Tsai YR, Chi MF, Chang LC, Li CF. An experience nursing a patient with dengue hemorrhagic fever. $\mathrm{Hu} \mathrm{Li} \mathrm{Za}$ Zhi. 2005;52(4):88-93.

53. Rusinger EZ, Fritzche C, Krause R, Krejs GJ. Diarrhea caused by primarily nongastrointestinal infections. Nat Clin Pract Gastroenterol Hepatol. 2005;2(5): 216-22.

54. Pancharoen C, Thiskayorn U. Dengue virus infection during infancy. Trans Royal Soc Trop Med Hyg. 2001;95(3): 307-8.

55. Seet RC, Ooi EE, Wong HB, Paton NI. An outbreak of primary dengue infection among migrant Chinese workers in Singapore characterized by prominent gastrointestinal symptoms and a high proportion of symptomatic cases. J Clin Virol. 2005;33(4):336-40.

56. Rongrungruang Y, Leelarasamee A. Characteristics and outcomes of adult patients with symptomatic dengue virus 
infections. J Infect Dis Antimicrol Agents. 2001;18(1):19-23.

57. Neo HY, Wong RC, Seto KY, Yip TW, Yang $\mathrm{H}$, Ling LH. Noncompaction cardiomyopathy presenting with congestive heart failure during intercurrent dengue viral illness: importance of phenotypic recognition. Int J Cardiol. 2006; 107(1):123-5.

58. Young P. Detection of NS1 from dengue virus: basis for early diagnosis and a prognostic marker of disease progression (1). Dengue diagnostics: proceedings of an international workshop. Geneva: WHO/TDR; 2004. (TDR/ IRM/DIAG/DEN/05.1).

59. Flammand M, Alcon-La Poder S, Drouet MT, Sivard P. Detection of NS1 from dengue virus: basis for early diagnosis and a prognostic marker of disease progression (2). Dengue diagnostics: proceedings of an international workshop. Geneva: WHO/TDR; 2004. (TDR/IRM/ DIAG/DEN/05.1).

60. Kittigul L, Suankeow K, Sujirarat D, Yoksan S. Dengue hemorrhagic fever: knowledge, attitude and practice in Ang Thong Province, Thailand. Southeast Asian J Trop Med Public Health. 2003; 34(2):385-92.

61. Díaz Quijano FA, Martínez Vega RA, Villar Centeno LA. Early indicators of severity in dengue virus infection. Enferm Infecc Microbiol Clin. 2005;23(9): 529-32.
62. Ahluwalia G, Sharma SK. Dengue: current trends and challenges. An Indian perspective. J Assoc Physicians India. 2004;52:561-3.

63. Magpusao NS, Monteclar A, Deen JL. Slow improvement of clinicallydiagnosed dengue hemorrhagic fever case fatality rates. Trop Doct. 2003;3(3): 156-9.

64. Kalayanarooj S. Standardized clinical management: evidence of reduction of dengue hemorrhagic fever case-fatality rate in Thailand. Dengue Bull. 1999;23: $10-7$.

65. Harris E, Pérez L, Pheres CR, Pérez ML, Idiaquez $\mathrm{W}$, Rocha $\mathrm{C}$, et al. Fluid intake and decreased risk for hospitalization for dengue fever, Nicaragua. Emerg Infect Dis. 2003 9(8):1003-6.

66. Halstead SB. More dengue, more questions. Emerg Infect Dis. 2005;11(5): 740-1.

67. Shah I, Deshpande GC, Tardeja PN. Outbreak of dengue in Mumbai and predictive markers for dengue shock syndrome. J Trop Pediatr. 2004;50(5): 301-5.

68. Carlos CC, Oishi K, Cinco MT, Mapua $\mathrm{CA}$, Inoue S, Cruz DJ, et al. Comparison of clinical features and hematologic abnormalities between dengue fever and dengue hemorrhagic fever among children in the Philippines. Am J Trop Med Hyg. 2005;73(2):435-40.

69. Méndez A, González G. Dengue hemorrágico en niños: diez años de experiencia clínica. Biomédica. 2003;23:180-93.
70. Wills BA, Nguyen MD, Ha TL, Dong TH, Tran TN, Le TT, et al. Comparison of three fluid solutions for resuscitation in dengue shock syndrome. N Eng J Med. 2005;353(9):877-89.

71. Ranjit S, Kissoon N, Jayskumar J. Aggressive management of dengue shock syndrome may decrease mortality rate: a suggested protocol. Pediat Crit Care Med. 2005;6(4):412-9.

72. Shann F. Severe dengue: coming soon to a pediatric intensive care unit near you? Pediatr Crit Care Med. 2005;6(4): 490-2.

73. Martínez E, Velázquez JC. Dengue. En: Ruza F, ed. Tratado de cuidados intensivos pediátricos. 3. ${ }^{\mathrm{a}}$ ed. Madrid: Capitel-Norma Ediciones; 2002. Pp. 1760-4.

74. Navarrete Espinosa J, Gómez Dantes H, Celis Quintal JG, Vázquez Martínez JL. Clinical profile of dengue hemorrhagic fever cases in Mexico. Salud Publica Mex. 2005;47(3):193-200.

75. World Health Organization. Dengue hemorrhagic fever: diagnosis, treatment, prevention and control. 2nd ed. Geneva: WHO; 1997.

76. Wiwanitkit V, Manusvanich P. Can hematocrit and platelet determination on admission predict shock in hospitalized children with dengue hemorrhagic fever? A clinical observation from a small outbreak. Clin Appl Thromb Hemost. 2004;10(1):65-7.

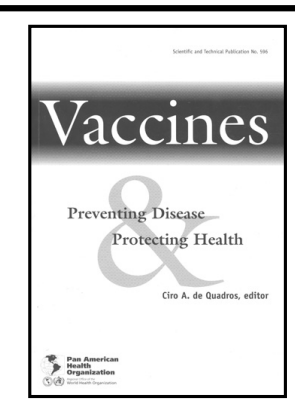

\section{Vaccines: Preventing Disease and Protecting Health IS NOW AVAILABLE IN ELECTRONIC FORMAT}

In this book you will find information about how vaccines have improved the health of the world's populations. The book relates successful efforts to fight disease with vaccines, including the eradication of polio from the Americas, and the potential contribution of new measles vaccine formulations to reducing measles mortality worldwide. It also looks at the challenges posed in using vaccines to cope with emerging and re-emerging diseases, such as HIV/AIDS, and in the fight against bioterrorism.

Electronic Version: 2004, 412p., ISBN 927511596 6, Order code: SP-E 596, Price: US\$40.00

To access the electronic version, follow these three simple steps:

1. Visit the site: www.ingentaselect.com

2. Select Pan American Health Organization in the Browse by publisher option

3. Select Vaccines: Preventing Disease and Protecting Health

Or go directly to PAHO publications by visiting:

http://www.ingentaselect.com/pubjournals.htm?pb=paho 